\title{
Variation of Terpenoid Flavor Odorants in Bran of Some Black and White Rice Varieties Analyzed by GC $\times$ GC-MS
}

\author{
Watcharapong Chumpolsri ${ }^{1}$, Nataporn Wijit ${ }^{1}$, Pittayaporn Boontakham ${ }^{1}$, Piyarat Nimmanpipug ${ }^{1}$, \\ Phumon Sookwong ${ }^{1}$, Suwaporn Luangkamin ${ }^{2}$, Sugunya Wongpornchai ${ }^{1, *}$ \\ ${ }^{1}$ Center of Excellence for Innovation in Chemistry and Department of Chemistry, Faculty of Science, Chiang Mai University, \\ Chiang Mai 50200 Thailand \\ ${ }^{2}$ Department of Chemistry, Faculty of Science at Si Racha, Kasetsart University, Si Racha Campus, Chonburi 20230 Thailand \\ *Corresponding author: scismhth@chiangmai.ac.th, sugunya.w@gmail.com
}

Received November 16, 2014; Revised February 08, 2015; Accepted February 12, 2015

\begin{abstract}
Among the total of 146 volatiles extracted by SPME from the headspace of some black and white rice bran samples, twenty-eight terpenoid odorants were acculately identified. Most of these terpenoids possess good aroma character and are varied among three groups of Thai rice; black glutinous, black non-glutinous, and white non-glutinous. Of these three groups, black non-glutinous rice contains the greater number of these monoterpenoids. However, the content of the major terpenoid odorants, which are limonene, trans- $\beta$-ocimene, $\beta$-cymene, and linalool, is the highest in the bran of white Thai jasmine rice, Khao Dawk Mali 105 (KDML 105). An herbaceous odorant, myrcene, occurs in the bran of all black rice varieties but not in the bran of white rice. The flavor type of rice bran using these rice bran terpenoids was successfully classified using the chemometric principle component analysis method.
\end{abstract}

Keywords: aroma, terpenoids, solid phase microextraction, two-dimensional gas chromatography-mass spectrometry, GC×GC-MS, chemometrics, PCA

Cite This Article: Watcharapong Chumpolsri, Nataporn Wijit, Pittayaporn Boontakham, Piyarat Nimmanpipug, Phumon Sookwong, Suwaporn Luangkamin, and Sugunya Wongpornchai, "Variation of Terpenoid Flavor Odorants in Bran of Some Black and White Rice Varieties Analyzed by GC×GC-MS.” Journal of Food and Nutrition Research, vol. 3, no. 2 (2015): 114-120. doi: 10.12691/jfnr-3-2-7.

\section{Introduction}

Rice (Oryza sativa L.), a major food commodity in most part of the world, has been studied the most among all cereal grains for its nutrients and phytochemicals, especially those related to eating quality. The type of rice that generates a pleasant odor during or after cooking is generally preferred by consumers. This pleasant aroma adds value to the rice and classifies it as fragrant rice. The characteristic aroma of most fragrant rice varieties is due mainly to the presence of a key odorant, 2-acetyl-1pyrroline (2AP) [1]. This impact aroma compound has its low threshold value at $0.1 \mathrm{ppb}$ in water [2]. It occurs naturally in rice as well as in some other plants, such as pandan leaves and bread flowers [3,4]. Although higher concentrations of 2AP in grains of fragrant rice distinguish it from non-fragrant rice, a number of other odorants are genetically synthesized and accumulated in rice grain. This has resulted in some rice varieties with different aroma qualities. Examples are the Thai black glutinous rice varieties, known as Kao Niow Dam. In Thailand, this type of rice is mostly consumed in sweetened form as dessert because it has a very rich nutty flavor. In recently years, according to the therapeutic functions $[5,6]$ of the black rice attributable to its pigmented phytochemicals, some non-glutinous Thai black rice varieties had been produced and had gained increasing popularity as a staple food replacing white rice. The popular non-glutinous black rice varieties available in the Thai rice market that could demand higher price are also those that possess slightly sweet odor.

Since characteristic flavor of black rice varieties plays an important role in the rice's reputation and acceptance by customers, this research explored those volatile aroma compounds that play an important role in the odor of black rice. In our preliminary experiment, contour plots of overall volatile component profiles of some black and white rice bran samples obtained by comprehensive twodimensional gas chromatography-mass spectrometry (GC $\times$ GC-MS) revealed many differences in the region of terpenoid components. Further investigation identified and quantified these rice bran terpenoid odorants, comparing them among groups of black glutinous, black nonglutinous, and white jasmine rice. Thus, a good understanding of some aroma-active compounds that are predominant in the characteristic flavor of black rice can now be used to increase the development program of aromatic rice. 


\section{Materials and Methods}

\subsection{Rice Samples}

Nine varieties of rice were used in this study and were divided into three groups: white non-glutinous KDML 105 and Sin Lek; black non-glutinous Hom Nin, and BT No.3; and black glutinous Samoeng, Black Rose, BGMSN, 16038, and BD. Sin Lek and BT No.3 are crosses between KDML 105 and black non-glutinous Hom Nin. This crossing resulted in a selected line, BD. All white and black non-glutinous rice samples and the black glutinous rice $\mathrm{BD}$ were collected from the experimental field in Kasetsart University, Kamphaengsaen Campus, Nakorn Pathom Province in central Thailand. The other black glutinous rice samples were obtained from the experimental field of Chiang Mai University, Chiang Mai Province in northern Thailand. The rice samples, all with moisture contents of approximately $14 \%$ (wb), were stored in gunny sacks and kept in a control room at $15^{\circ} \mathrm{C}$. On the day of the experiment, each rice sample was dehusked and milled, using a local milling system (Natrawee Technology, Chachoengsao, Thailand) for $40 \mathrm{~s}$ to obtain approximately $12-15 \%(\mathrm{w} / \mathrm{w})$ of fresh rice bran.

\subsection{Chemicals}

The mixtures of $\mathrm{C}_{8}$ to $\mathrm{C}_{22} n$-alkanes used in GC-MS analysis for determination of the retention index, along with a set of standard terpenoids, $\alpha$-pinene, myrcene, $1,4-$ cineol, $\beta$-cymene, limonene, ocimene, $\gamma$-terpene, cislinalool oxide, trans-linalool oxide and linalool, were purchased from Merck (Darmstadt, Germany).

\subsection{SPME of Rice Bran Headspace Volatiles}

The SPME device used was a manual sampling device equipped with a polydimethylsiloxane (PDMS) fiber (Supelco, Bellefonte, PA, USA) $1 \mathrm{~cm}$ long and $100 \mu \mathrm{m}$ thick. This fiber was heated in a GC injection port set at $250^{\circ} \mathrm{C}$ for $30 \mathrm{~min}$ in order to get rid of contaminants and moisture. The SPME parameters, which were equilibration time and temperature, absorption time and temperature as well as amount of rice bran sample were optimized in order to obtain a good sensitivity of volatile terpenoid odorants. Each sample vial, containing 15.0 g of rice bran, was heated at $100^{\circ} \mathrm{C}$ for 35 min before the fiber was exposed for $30 \mathrm{~min}$ at $100^{\circ} \mathrm{C}$ to the headspace above rice bran.

\subsection{GC-MS and GC $\times$ GC-MS operation conditions}

The GC-MS system utilized was an Agilent 6890 and HP 5973 mass-selective detector (Agilent Technologies, Polo Alto, CA, USA) equipped with a split-splitless injector operating in the splitless mode. A HP-5 capillary column (J\&W Scientific, Folsom, CA) $30 \mathrm{~m} \times 0.25 \mathrm{~mm}$ i.d., with a thickness of $0.25 \mu \mathrm{m}$ was used. The temperature program of the GC was as follows: initial column temperature was $45^{\circ} \mathrm{C}$ for $4 \mathrm{~min}$, then increase at a rate of $2{ }^{\circ} \mathrm{C} / \mathrm{min}$ to $100{ }^{\circ} \mathrm{C}$, and finally increase to $250{ }^{\circ} \mathrm{C}$ at a rate of $3{ }^{\circ} \mathrm{C} / \mathrm{min}$. The temperature of both the injector and detector was $250{ }^{\circ} \mathrm{C}$. Pure helium gas at a flow rate of $1 \mathrm{~mL} / \mathrm{min}$ was used as the carrier gas. The GC splitless valve was set to open after 0.2 min of injection. The MS was operated in the electron impact mode with electron energy of $70 \mathrm{eV}$, ion source temperature at $230{ }^{\circ} \mathrm{C}$, quadrupole temperature at $150{ }^{\circ} \mathrm{C}$, and scan mass range of $\mathrm{m} / \mathrm{z}$ 29-550. The GC-MS transfer line was set to $280{ }^{\circ} \mathrm{C}$. Volatile component identification was mainly done by matching the mass spectrum of each component with reference spectra in the NIST 11 Mass Spectral Library. In addition, published Kováts indices and retention times of the standards, for some available compounds, were used to aid structural confirmation. Quantitative analysis of each volatile component was performed by peak area normalization measurement and relative quantities of volatiles contained in each rice bran sample extract were in percent.

The GC $\times$ GC-MS operation were done using the GC model HP 6890 which was retrofitted with equipped with a secondary oven and non-moving quad-jet dual stage modulator. The GC $\times$ GC was coupled to a Pegasus (III) time-of-flight mass spectrometer (LECO Corporation, St. Joseph, MI, USA). The LECO ${ }^{\circledR}$ ChromaTOFTM software (version 2.00) was used to operate the GC×GC-TOF-MS system, which was operated at $100 \mathrm{~Hz}$ data acquisition. The second column was connected to the TOF-MS instrument (Leco, St. Joseph, MI, USA) via an empty deactivated column $(0.5 \mathrm{~m} \times 0.25 \mathrm{~mm}$ i.d. $)$. The columns and the empty deactivated column were connected by SGE unions using SilTite metal ferrules (Austin, Texas, USA) for $0.10-0.25 \mathrm{~mm}$ i.d. GC columns. Three set of GC $\times$ GC column were used. The non-polar/polar column set comprised a HP-5 capillary column $(30 \mathrm{~m} \times 0.25 \mathrm{~mm}$ i.d., $0.25 \mu \mathrm{m}$ thickness) and a BP-20 capillary column (1.0 $\mathrm{m} \times 0.10 \mathrm{~mm}$ i.d., $0.10 \mu \mathrm{m}$ thickness). The polar/non-polar column set comprised a Solgel wax capillary column (30 $\mathrm{m} \times 0.25 \mathrm{~mm}$ i.d., $0.25 \mu \mathrm{m}$ thickness) and a BP-1 capillary column (1.0 $\mathrm{m} \times 0.10 \mathrm{~mm}$ i.d., $0.10 \mu \mathrm{m}$ thickness). The third set combined an enantioselective first dimension column, which was a EtTBS- $\beta$ CD capillary column (30 m $\times 0.25 \mathrm{~mm}$ i.d., $0.25 \mu \mathrm{m}$ thickness) to a BP-20 capillary column $(1.0 \mathrm{~m} \times 0.10 \mathrm{~mm}$ i.d., $0.10 \mu \mathrm{m}$ thickness $)$. The GC condition was the same as previous. All columns were available from SGE International (Ringwood, Australia) except A HP-5 column from J\&W Scientific, Folsom, CA and the enantioselective EtTBS- $\beta \mathrm{CD}$ column from MeGA (Milan, Italy).

\subsection{Data Conversion and Principle Component Analysis}

Chromatographic data from GC×GC-MS were exported in an ASCII file format (*.csv files) and were then transformed as contour plots based on the modulation frequency and sampling rate using the Agilent Chemstation software version B.01.03[204]. For data analysis of total peak height and area, the relevant files were exported as *.csv integration files prior to analysis using a Matlab program version 6.1, which summed the peak pulses for each component to give the total area for that component. Principal component analysis (PCA) was done mainly on odor-active compounds by statistical package program that evaluated the relationships in terms of correlation among groups of multivariate data. The PCA output consisted of loading plots to explain the cluster separation between different samples. 
Table 1. Headspace terpenoids, their odor descriptions, and relative contents in bran samples of three groups of rice varieties

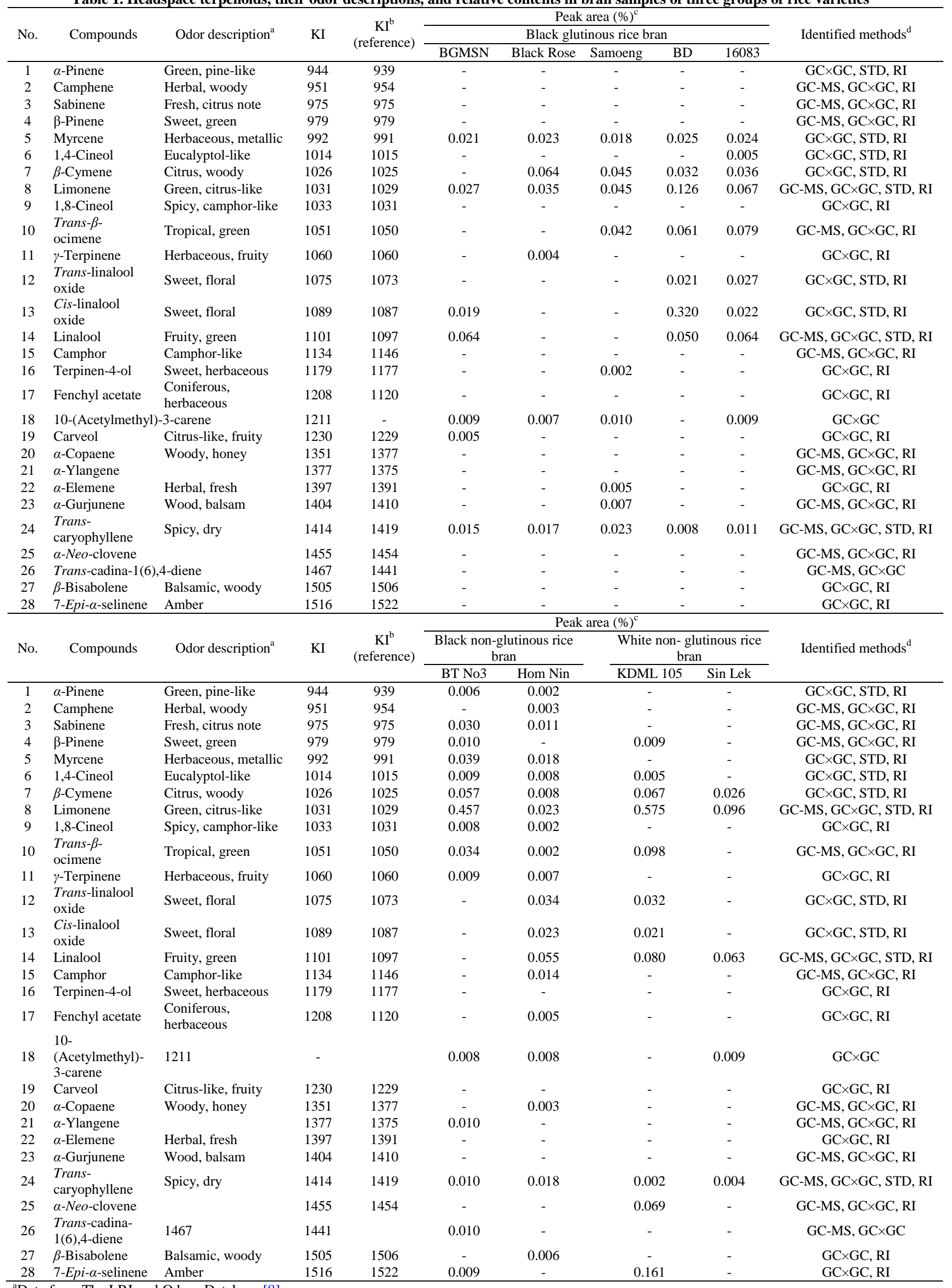

${ }^{\mathrm{a}}$ Data from The LRI and Odour Database [8]

bata from Adams [7] and The LRI and Odour Database [8]

'Obtained from triplicate analyses

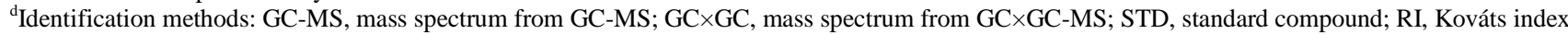




\section{Results and Discussion}

\subsection{Relative Contents of Terpenoid Odorants in Headspace of Rice Brans}

The headspace volatiles of the rice bran samples extracted by SPME were preliminary separated and identified by GC-MS. Apart from the mass spectral comparison with those compiled in the mass spectral library software, Kovát index (KI) matching was performed [7]. At least 103 rice bran headspace volatiles were identified among the three groups of rice samples.

However, structural confirmation of those active odorants in groups of monoterpenoids was obscure due to the high complexity of volatile components accumulated in the rice brans. Thus, the use of GC $\times \mathrm{GC}-\mathrm{MS}$, where many co-eluted peaks from 1D-GC could be entirely resolved on the 2D column was required. In this study, three GC $\times$ GC column combination sets were investigated. These were the conventional non-polar/polar combination (HP5/BP20), an inverse phase combination (Solgel wax/BP1) and a combination applying an enantioselective first dimension column (EtTBS- $\beta$ CD/BP20).

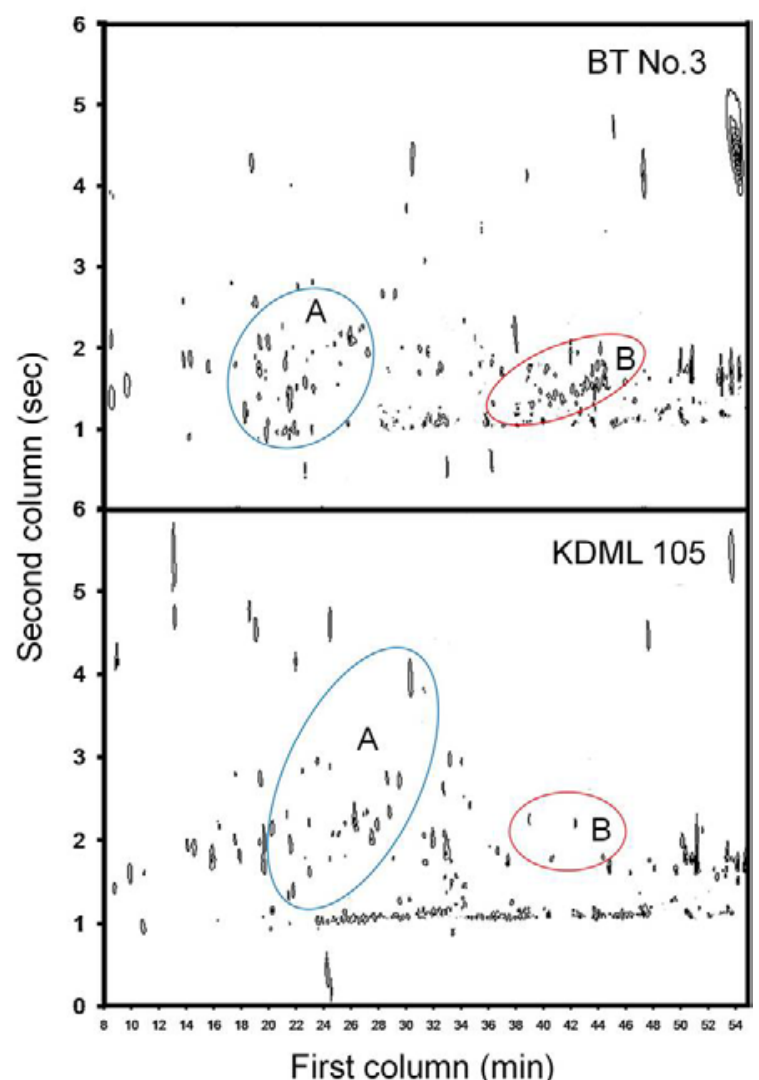

Figure 1. GC $\times$ GC-MS contour plots of black BT No.3 and white KDML 105 rice bran headspace volatiles extracted by SPME: (A) monoterpenes and oxygenated monoterpenoids and (B) sesquiterpenes

Although an enantioselective column is a good choice for the separation of essential oil isomeric components, a better resolution leading to an increased number of resolved components was observed using non-polar/polar column set. The separation using the polar/non-polar column set showed some broadened peaks, component wrap-around, and the tailing of some components. Thus, the HP5/BP20 column set was selected to achieve better separation and accurate identification of the rice bran volatiles. Some minor components, which were not apparent in the first column in GC-MS, were spread out within the second dimension according to an increase in resolution and sensitivity of the $\mathrm{GC} \times \mathrm{GC}$. The improved separation of the modulated peaks in the second dimension of $\mathrm{GC} \times \mathrm{GC}-\mathrm{MS}$ facilitated more accurate identification due to higher and more precise matched mass spectra. As a result, 43 more constituents were added to those obtained by GC-MS.

Figure 1 shows contour plots of overall rice bran volatiles with emphasis on those two groups of terpenoids separated in their positions on the 2D plane when the HP5/BP20 column set was used. Monoterpenoids, and sequiterpenoids are clearly separated into two regions, A and $\mathrm{B}$, respectively. $\mathrm{GC} \times \mathrm{GC}$ profiles of the rice terpenoids obtained from the nine rice bran samples are rather different and do not show the characteristic of any groups of rice samples. However, the well-resolved $\mathrm{GC} \times \mathrm{GC}$ peaks in these terpenoid regions unambiguously confirmed the identity of these rice terpenoids and made their relative quantity more accurate.

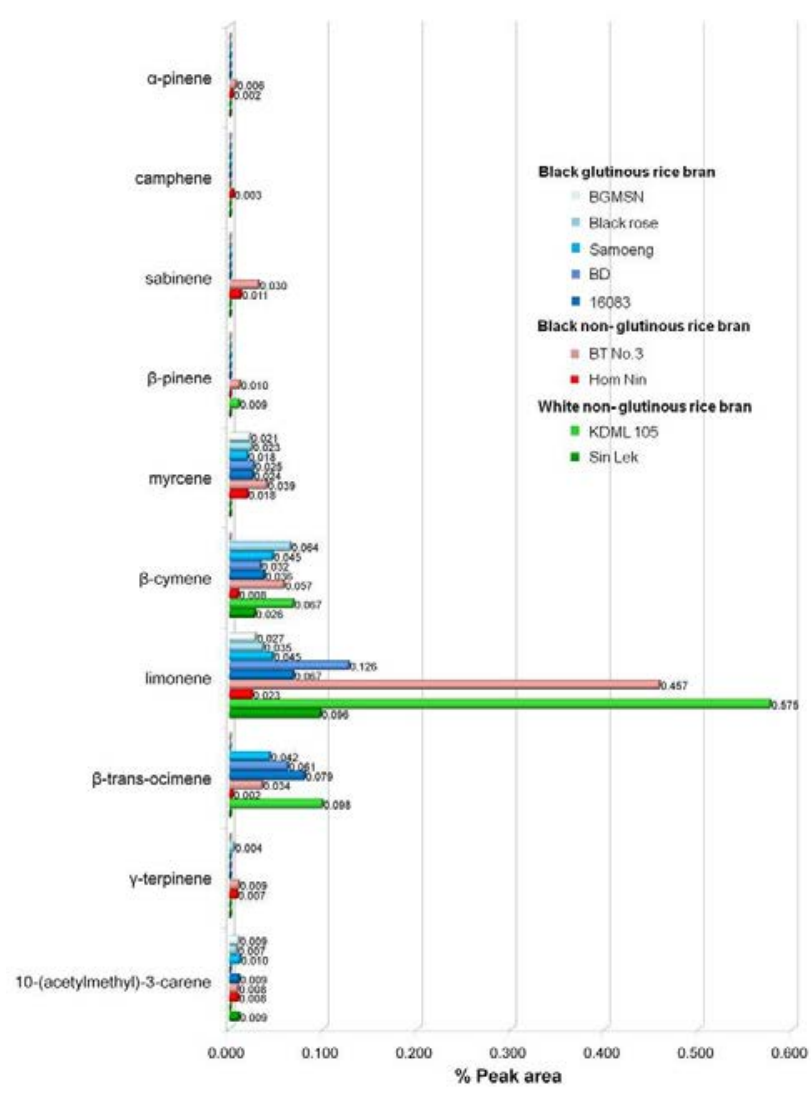

Figure 2. Comparison of monoterpene odorants in bran samples of three groups of rice varieties

Among the three groups of rice samples, there were 19 monoterpenoids and 9 sesquiterpenoids present in reasonable amounts (Figure 2 and Figure 3). The relative content of the monoterpenoids was much higher, on average, than that of the sesquiterpenoids. The comparison of relative contents of monoterpenoids and sesquiterpenoids in rice bran headspace indicated higher numbers of these terpenoids in the bran of black non-glutinous rice than in the bran of the other rice samples. The major terpenoids, which were limonene, trans- $\beta$-ocimene, $\beta$-cymene, linalool, 
and myrcene, were widely distributed among the three groups of the rice samples. However, some minor terpenoids were found only in particular rice varieties.

Odorants in groups of terpenoids, especially the monoterpenoids and sesquiterpenoids, play an important role in aroma quality of foods. Limonene is the prominent monoterpene odorant and all rice bran samples used in this study contained limonene. This monoterpene has a citruslike odor and had previously been reported in the headspace extracts of two fragrant rice (Oryza sativa L.) cultivars grown in South China [9], cooked black rice [10] and steam distillate extract of some cooked white rice $[11,12]$. The highest content of limonene, $\beta$-cymene, and trans- $\beta$-ocimene occurred in the bran of white Jasmine rice KDML 105. These monoterpenes also were found in most of the rice bran samples, while myrcene, a key intermediate in the production of several fragrances, was in all black rice bran samples but not in the bran of the two white rice samples. This monoterpene could, therefore, be considered as a characteristic odorant that give a herbaceous odor to the overall aroma of black rice.



Figure 3. Comparison of some oxygenated monoterpenoids and sesquiterpene odorants in bran samples of three groups of rice varieties

In the group of oxygenated monoterpenoids, linalool, and its metabolic products, cis- and trans-linalool oxide, were detected as the major oxygenated monoterpenes. However, they occurred only in some bran samples of the three rice variety groups. Rice varieties whose brans contained all these major oxygenated monoterpenoids were black glutinous BD and 16083, black non-glutinous
Hom Nin, and white KDML 105 rice. The relative content of some major monoterpenoids, such as limonene and cislinalool oxide, in a rice variety could be approximately 10 times greater than in other rice varieties. The sweet, fruity, and floral odors of these monoterpenoids in conjunction with their high volatility gives them an important role in rice aroma quality. Trans-caryophyllene, a spicy and dry odorant of clove oil [13], was found as a prominent sesquiterpene. It occurred in the bran of all rice samples, although its relative amount was much lower than that of 7-epi- $\alpha$-selinene and $\alpha$-neo-carvene, which were found mainly in the bran of KDML 105.

Most of the minor terpenoids occur in only a few rice varieties. Terpinen-4-ol (sweet and herbaceous), $\alpha$-gurjunene, and $\alpha$-elemene were found only in the bran of the black glutinous rice. Other terpenoids occur only in the black non-glutinous varieties. These being $\alpha$-pinene, camphene, sabinene, 1,8-cineol, camphor, menthol, estrageol, fenchyl acetate, $\alpha$-copaene, $\alpha$-ylangene, trans-cadina-1(6),4-diene, and $\beta$-bisabolene. Most of these terpenoids were previously identified in a variety of natural and processed rice, including California Brown Pearl rice [14], hydrodistillate extracts of red and black Japanese rice brans [15,16], and extracts of Basmati, Jasmine, Australian fragrant, and non-fragrant rices [17].

A major caraway odorant, $\alpha$-neo-carvene, was only detected in bran of KDML 105 white rice. Sabinene, which was reported to be a main component in the essential oils of marjoram [18], was found only in the black non-glutinous rice bran, such as BT No.3 and Hom Nin. $\beta$-Cymene was found in all rice bran samples except BGMSN, the highest amount being in the bran of Samoeng black rice. This terpenoid is a representative of a small group of aromatic compounds that are produced in nature from isoprene units being formed by dehydrogenation of $\alpha$-terpinene. A major eucalyptus oil component, 1,8-cineol, has a fresh camphor-like and spicy odor. It was found only in BT No.3 and Hom Nin black non-glutinous rice bran. Carveol, which is the metabolic products of limonene $[19,20,21]$, was found only in BGMSN.

The bran of black non-glutinous rice, such as Hom Nin, contains $\alpha$-pinene. $\beta$-Pinene occurs in the bran of Jasmine KDML 105 white non-glutinous rice. The presence of these two monoterpenes in BT No.3, which is the selected cross between Hom Nin and Jasmine KDML 105, confirms their genetic relationship. Fenchyl acetate was found only in Hom Nin, the same as camphor and camphene. Camphor has a pungent odor and occurs in camphor oil, as does camphene. These monoterpenoids are naturally produced from the same precusor, $\alpha$-terpinyl cation $[19,20]$. They were found only in the bran of Hom Nin black non-glutinous rice. Additionally, vanillin, a phenolic aldehyde having an important flavoring character, was found in all of the rice bran samples. Its highest content was in bran of Jasmine KDML 105 rice.

\subsection{Classification of Rice Bran Flavor Types by Terpenoid Components}

PCA analysis was performed on terpenoid groups of odorants. The plot of this analysis using average peak area $(n=3)$ in each rice bran sample extract for the 28 terpenoids in Table 1 is shown in Figure 2A and Figure 
2B. The first two principal components accounted for $57.3 \%$ of total variance [PC 1 (37.3 \%) + PC 2 (20.0\%)]. Fig. 2A shows that rice bran can be classified into three groups. BT No.3, which is a crossbreed between KDML 105 and black non-glutinous Hom Nin, was segregated in the same group with KDML 105. This indicated that variation of terpenoid components in BT No.3 obtained from gene of KDML 105. BGMSN, Sin Lek, Samoeng, Black Rose, 16083 and BD were segregated in the other group, whereas Hom Nin was segregated in the last group. The main terpenoid components having an influence on PC1 were $\alpha$-pinene, camphene, sabinene, myrcene, 1,4cineol, $\beta$-cymene, 1,8-cineol, $\gamma$-terpinene, trans-linalool oxide, cis-linalool oxide, linalool, camphor, fenchyl acetate, 10 -(acetylmethyl)-3-carene, $\alpha$-copaene, transcaryophyllene, $\beta$-bisabolene. This indicates that these terpenoids are derived either exclusively or predominately from BGMSN, Sin Lek, Samoeng, Black Rose, 16083 and $\mathrm{BD}$ rice. Based on PC 2, the groups were separated by $\beta$ pinene, limonene, $\alpha$-ylangene, trans-cadina-1(6),4-diene and 7-epi- $\alpha$-selinene. Therefore, classification of rice flavor types using PCA method can be achieved by using these rice bran terpenoids.


Figure 4. PCA plots from nine rice bran samples; (A) the separation of different rice bran based on terpenoid compounds and (B) the distribution of 28 terpenoid compounds (loadings) in relation to different rice bran. Numbers correspond to compounds listed in Table 1

\section{Conclusion}

The increased sensitivity and resolution obtained by GC $\times$ GC-MS in the separation of rice bran volatiles extracted by SPME accurately revealed 20 monoterpenoids and 9 sesquiterpenoid odorants. The greater number of these terpenoids occur in bran of the black non-glutinous rice group. Although some major terpenoids were widely distributed among the three groups of the rice samples, the highest content of these major terpenoids occurred in bran of the white Thai jasmine rice. An herbaceous odorant, myrcene, was found in bran of all black rice varieties but not in white rice bran. This monoterpene could, therefore, be considered as a characteristic odorant of black rice. Also, the use of these rice bran terpenoids by PCA method could clearly classifies samples of the nine rice varieties into three rice flavor types.

\section{Acknowledgement}

We thank Drs. Apichart Vanavichit and Sakda Jongkaewwattana for their help and support with the rice samples and the Center of Excellence for Innovation in Chemistry (PERCH-CIC), and the Higher Education Research Promotion and National Research University Project of Thailand, Commission on Higher Education, Ministry of Education, for their partial supports. Full financial support was given to Watcharapong Chumpolsri and Sugunya Mahatheeranont from the Thailand Research Fund through the Royal Golden Jubilee Ph.D. Program (Grant No. PHD/0015/2550).

\section{References}

[1] Buttery, R.G., Ling, L.C., and Juliano, B.O., "2-Acetyl-1-pyrroline: an important aroma component of cooked rice". Chemistry and Industry, 1982. 23: 958-959.

[2] Buttery, R.G., Turnbaugh, J.G., and Ling, L.C., "Contribution of volatiles to rice aroma". Journal of Agricultural and Food Chemistry, 1988. 36(5): 1006-1009.

[3] Buttery, R.G., Ling, L.C., Juliano, B.O., and Turnbaugh, J.G., "Cooked rice aroma and 2-acetyl-1-pyrroline". Journal of Agricultural and Food Chemistry, 1983. 31: 823-828.

[4] Wongpornchai, S., Sriseadka, T., and Choonvisase, S., "Identification and quantitation of the rice aroma compound, 2acetyl-1-pyrroline, in bread flowers (Vallaris glabra Ktze)". Journal of Agricultural and Food Chemistry, 2003. 51(2): 457462.

[5] Boo, H.O., Heo, B.G., and Gorinstein, S., "Analytical methods for enzyme and DPPH radical scavenging activities of natural pigments from some plants". Food Analytical Methods, 2012. 5(6): 1354-1361.

[6] Leardkamolkarn, V., Thongthep, W., Suttiarporn, P., Kongkachuichai, R., Wongpornchai, S., and Wanavijitr, A., "Chemopreventive properties of the bran extracted from a newlydeveloped Thai rice: The Riceberry". Food Chemistry, 2011. 125(3): 978-985.

[7] Adams, R.P., Identification of essential oil components by gas chromatography/quadrupole mass spectroscopy. (essential oils). Vol. 3. 2001, Illinois USA: Allured Publishing Corporation. 9-456.

[8] Mottram, R. The LRI \& Odour Database, Research Group, School of Food Biosciences, University of Reading, UK. [cit. 1 April 2014]. 2005; Available from: http://www.odour.org.uk/index.html.

[9] Goufo, P., Duan, M., Wongpornchai, S., and Tang, X., "Some factors affecting the concentration of the aroma compound 2acetyl-1-pyrroline in two fragrant rice cultivars grown in South China". Frontiers of Agriculture in China, 2010. 4: 1-9.

[10] Yang, D.S., Lee, K.S., Jeong, O.Y., Kim, K.J., and Kays, S.J., "Characterization of volatile aroma compounds in cooked black rice". Journal of Agricultural and Food Chemistry, 2008. 56(1): 235-240.

[11] Yajima., I., Yanai, T., and Nakamura, M., "Volatile flavour components of cooked rice." Agricultural and Biological Chemistry, 1978. 42: 1229-1233.

[12] Yajima, I., Yanai, T., and Nakamura, M., "Volatile flavor components of cooked kaorimai (scented rice, O. sativa japonica)". Journal of Agricultural and Biological Chemistry, 1979. 43: 24252430 . 
[13] Ghelardini, C., Galeotti, N., Di Cesare Mannelli, L., Mazzanti, G., and Bartolini, A., "Local anaesthetic activity of betacaryophyllene". Il Farmaco, 2001. 56: 387-389.

[14] Bullard, R.W. and Holguin, G., "Volatile components of unprocessed rice (Oryza sativa L.)". Agricultural and Food Chemistry, 1977. 25(1): 99-103.

[15] Sukhonthara, S., Theerakulkait, C., and Miyazawa, M. "Characterization of volatile aroma compounds from red and black rice bran". Journal of Oleo Science, 2009. 58(3): 155-161.

[16] Tsugita, T., Kurata, T., and Fujimaki, M., "Volatile components in the steam distillate of rice bran: identification of neutral and basic compounds". Agricultural and Biological Chemistry, 1978. 42: 643-651.

[17] Widjaja, R., Craske, J.D., and Wootton, M., "Comparative studies on volatile components of non-fragrant and fragrant rices".
Journal of the Science of Food and Agriculture, 1996. 70(2): 151161.

[18] Reverchon, E., "Fractional separation of SCF extracts from marjoram leaves: Mass transfer and optimization". The Journal of Supercritical Fluids, 1992. 5(4): 256-261.

[19] Bouwmeester, H.J., Gershenzon, J., Konings, M.C.J.M., and Croteau, R., "Biosynthesis of the monoterpenes limonene andcarvone in the fruit of caraway". Plant Physiology, 1998. 117(3): 901-912.

[20] Degenhardt, J., Köllner, T.G., and Gershenzon, J., "Monoterpene and sesquiterpene synthases and the origin of terpene skeletal diversity in plants". Phytochemistry, 2009. 70(15-16): 1621-1637.

[21] Dewick, P.M., Medicinal Natural Products A Biosynthetic Approach 3rd Edition. Vol. 3. 2009, West Sussex, United Kingdom: John Wiley \& Sons Ltd. 193-206. 